UCRL-JC-134713

PREPRINT

\title{
A New Global Hydrogen Equation of State Model
}

David A. Young

This paper was prepared for submittal to the

IIth Topical Conference on Shock Compression of Condensed Matter

Snowbird, UT

June 27-July 2, 1999

June 25, 1999

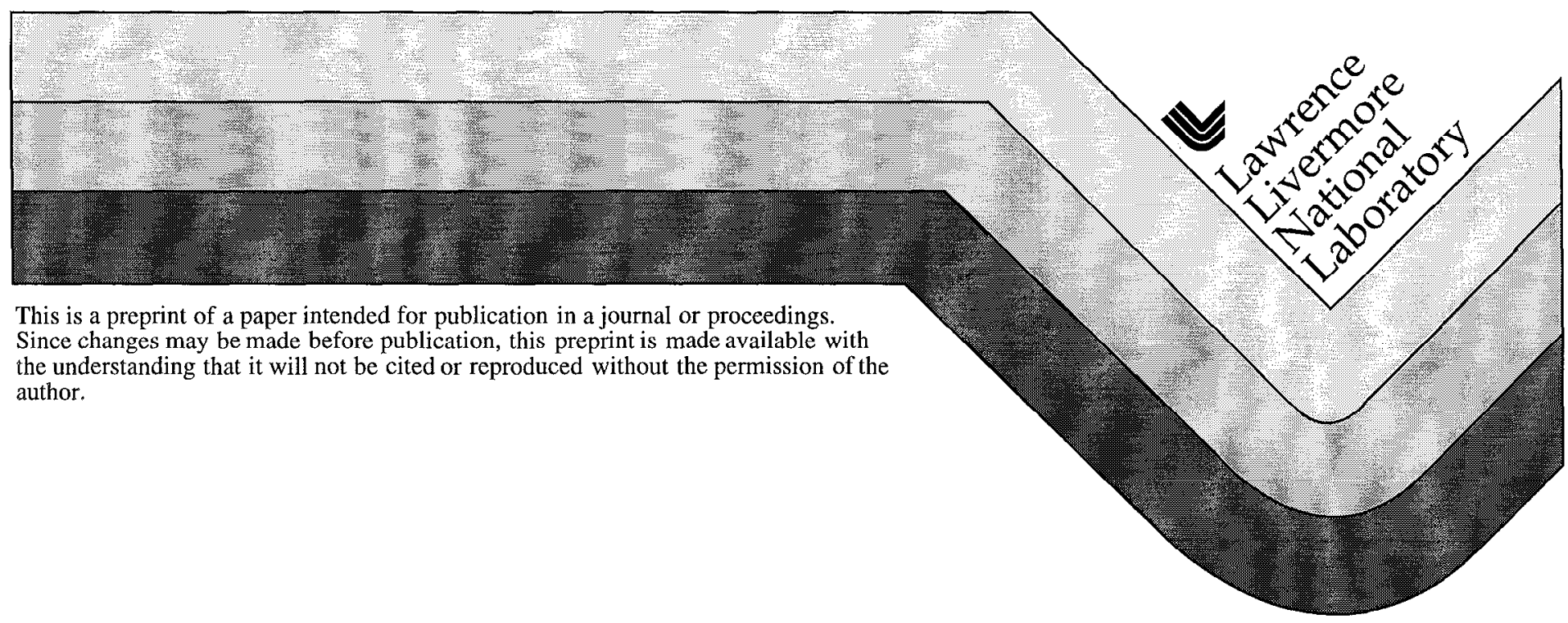




\section{DISCLAIMER}

This document was prepared as an acrount of work sponsored by an agency of the United States Government. Neither the United States Government nor the University of California nor any of their employees, makes any warranty, express or implied, or assumes any legal liability or responsibility for the accuracy, completeness, or usefulness of any information, apparatus, product, or process disclosed, or represents that its use would not infringe privately owned rights. Reference herein to any specific commercial product, process, or service by trade name, trademark, manufacturer, or otherwise, does not necessarily constitute or imply its endorsement, recommendation, or favoring by the United States Government or the University of California. The views and opinions of authors expressed herein do not necessarily state or reflect those of the United States Government or the University of California, and shall not be used for advertising or product endorsement purposes. 


\title{
A NEW GLOBAL HYDROGEN EQUATION OF STATE MODEL
}

\author{
David A. Young \\ Lawrence Livermore National Laboratory, P.O. Box 808, Livermore, California 94551 USA
}

\begin{abstract}
Simple statistical mechanics models have been assembled into a wide-range equation of state for the hydrogen isotopes. The solid is represented by an Einstein-Grüneisen model delimited by a Lindemann melting curve. The fluid is represented by an ideal gas plus a soft-sphere fluid configurational term. Dissociation and ionization are approximated by modifying the ideal gas chemical-equilibrium formulation. The $\mathrm{T}=0$ isotherm and dissociation models have been fitted to new diamond-anvil isotherm and laser-generated shock data. The main limitation of the model is in ionization at high compression.
\end{abstract}

\section{INTRODUCTION}

The design of laser-driven controlled fusion experiments relies heavily on hydrodynamic code simulations of the compression of microcapsules containing deuterium and tritium isotopes of hydrogen. Knowledge of the compressibility of these isotopes at high density and temperature is a requirement for the success of the simulations. The compressibility of matter over very wide ranges of density and temperature is embodied in a global equation of state (EOS), which is a representation of the pressure and energy as functions of density and temperature: $P(\rho, T)$ and $E(\rho, T)$.

In this paper I attempt to fit the existing experimental and theoretical data on compressed deuterium with a thermodynamically consistent global EOS, consisting of solid, liquid, and gas phases.

\section{THE DEUTERIUM COLD CURVE}

The assembly of the EOS model begins with the $\mathrm{T}=0$ static lattice isotherm (or "cold curve"), which determines the compressibility of the material over a wide range of pressure and temperature. There exists a number of experimental and theoretical data sets which allow a fairly accurate determination of the cold curve. Since most of the experiments have been performed on deuterium, I generate the EOS model for this isotope.

At low density and temperature, fluid deuterium consists of diatomic molecules interacting by weak van der Waals forces. This binding energy is experimentally known to be $\mathrm{E}_{\mathrm{c}}=1.104 \times 10^{10}$ $\mathrm{erg} /\left(\mathrm{mole}_{2}\right)$ [1]. If we use the convention that the energy of the $0 \mathrm{~K}$ solid at $\mathrm{P}=0$ is fixed at $E=0$, then $\mathrm{E}(\rho=0, \mathrm{~T}=0)=\mathrm{E}_{\mathrm{c}}$. Similarly, the normal $\mathrm{T}$ $=0, \mathrm{P}=0$ volume and bulk modulus are known: $\rho_{\mathrm{o}}$ $=0.202 \mathrm{~g} / \mathrm{cm}^{3}$ and $B_{0}=3.35 \mathrm{kbar}[1]$.

Using a simple "soft-sphere" formula [2] for the $\mathrm{T}=0$ energy and pressure, we can fit parameters to the conditions $P=0, E=0, B=B_{o}$ at $\rho=\rho_{o}$ and $T$ $=0$. This leaves one free parameter, which can be fitted to the liquid-vapor critical point.

For densities greater than $\rho_{0}$, the cold curve fitting is done in three sections. We can use a simple power series in the variable $\mu=\rho / \rho_{0}-1$ to fit experimental solid compression data to 1.2 Mbar [3].

An ultrahigh pressure static-lattice isotherm is available for monatomic metallic $\mathrm{H}$ from electron band-structure theory in the local-density approximation (LDA) [4]. These data have been computed to $10^{7} \mathrm{Mbar}$ or $10^{4} \mathrm{~g} / \mathrm{cm}^{3}$, and are fitted with a simple electron-gas-type function.

Because the LDA dala are for a monatomic lattice only, they do not match up precisely with the diatomic experimental data. Therefore I join the two fits with a cubic spline. No first-order phase 
change is assumed on pressure dissociation, and none is needed to fit the experimental and theoretical data. The cold energy is found by integrating the pressure function analytically. This 4-piece cold curve gives an accurate fit to the experimental and theoretical data, as shown in Fig. 1.

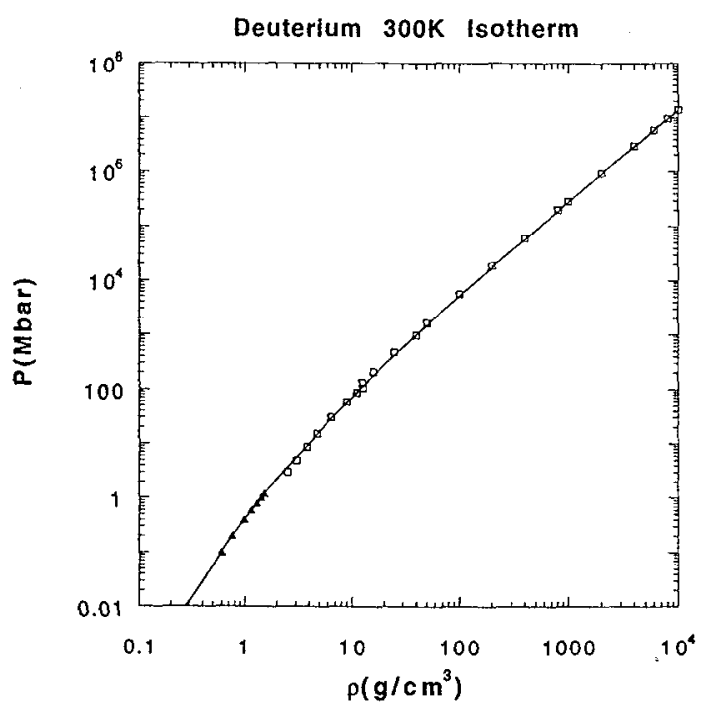

FIGURE 1. The model $300 \mathrm{~K}$ pressure isotherm for deuterium is shown as a smooth curve. The experimental data to $1 \mathrm{Mbar}$ are triangles and the higher pressure $\mathrm{T}=0 \mathrm{~K} \mathrm{LDA}$ theoretical data are shown as squares.

\section{SOLID AND FLUID MODELS}

The solid phase EOS consists of a GrüneisenEinstein model [5]. The Grüneisen function is assumed to decrease linearly with volume from $\gamma=$ 2.0 at normal solid volume to the one-component plasma limit of $\gamma=0.5$ at zero volume. At low density, the solid consists of diatomic molecules with internal rotational and vibrational degrees of freedom as well as center-of-mass lattice vibrational modes. At a critical dissociation density, the molecules dissociate to a monatomic lattice, and the model switches discontinuously to twice as many monatomic particles with lattice vibrational modes only. This dissociation density is consistent with the dissociation model which is used in the fluid state.

The melting temperature as a function of density is given by the approximate Lindemann law, which depends on the Grüneisen function. The fluid phase thermal EOS consists of an ideal gas of diatomic molecules, atoms, nuclei, and electrons in a mixture determined by a chemical equilibrium calculation, plus a nonideal configurational term. This latter term guarantees continuity with the solid free energy along the melting curve, as in the QEOS model [6]. There is no melting volume discontinuity in the model. The ideal gas partition functions for the monatonic and diatomic gases are $q_{\text {mon }}=q_{i} ; q_{d i a}=q_{t} q_{1} q_{v} q_{d}$, where the subscripts $t, r, v$, and $\mathrm{d}$ refer to translation, rotation, vibration, and dissociation.

The main constraint for the fluid model is to fit the experimental gas-gun $[7,8]$ and laser shock data $[9,10]$ as accurately as possible. This is a challenge, because the EOS models generated before the Nova shock data were released do poorly in comparison with the data [9]. The problem is the large Hugoniot density increase to about $1.05 \mathrm{~g} / \mathrm{cm}^{3}$ for deuterium, a compression ratio of more than 6 . This feature is undoubtedly due to the specific character of the molecular dissociation in hydrogen, which is quite different from other diatomics, such as nitrogen. I have fitted the model to the Nova shock data by allowing the dissociation energy to decrease with compression and for the diatomic fluid to have a large number of fictitious excited electronic states. These states are occupied without any corresponding increase in pressure, which allows the shock density to increase to the desired maximum value. The fit can be improved somewhat by adjusting the fluid configurational term. Above $2 \mathrm{Mbar}$, the shock density turns back toward the ideal gas limit of $4 \rho_{\text {in }}=0.68 \mathrm{~g} / \mathrm{cm}^{3}$ in deuterium. The comparison between the model and experiments is shown in Fig. 2.

It is interesting that a strong density dependence of the dissociation energy can introduce a phasetransition-like feature in the EOS. This is the "plasma phase transition" which is a hypothetical first-order fluid-fluid transition between the diatomic and monatomic states. Experimental evidence for this transition is still lacking.

The shock temperature has also been measured in both gas gun [8] and laser experiments [10], and the comparison is very good without making any additional model adjustments, as shown in Fig. 3. The dissociation region is clearly seen. I have also checked double-shock gas-gun data [7] and one sound-speed measurement in the shockcd state [11], and the agreement is good. This overall agreement suggests that fluid deuterium at temperatures below 


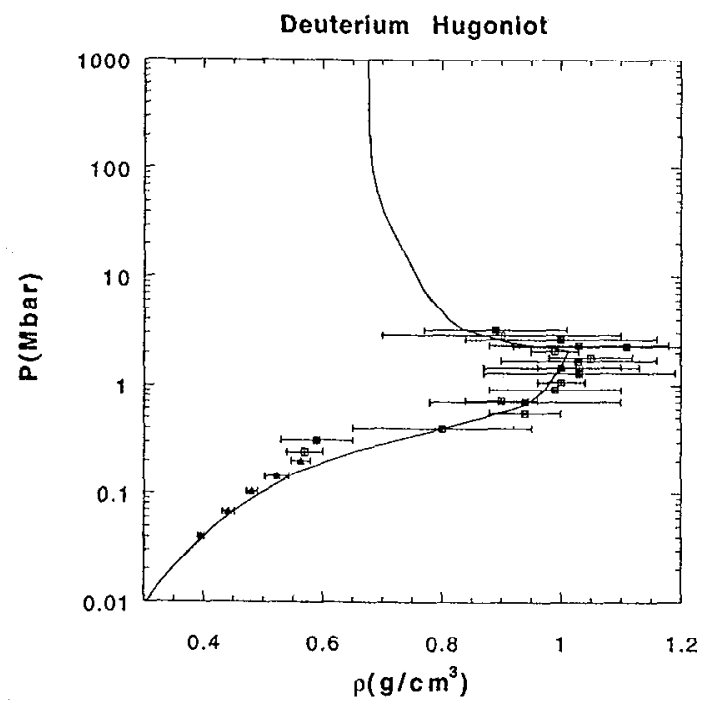

FIGURE 2. The deuterium pressure-density shock Hugoniot. The model prediction is shown as a smooth curve, the gas-gun data are triangles, and the Nova experimental data are squares.

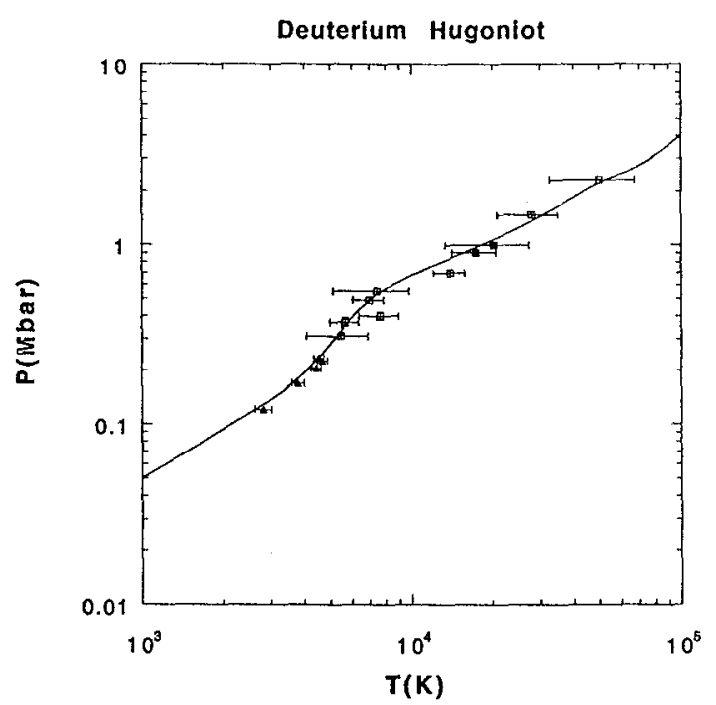

FIGURE 3. The deuterium pressure-temperature shock Hugoniot. The model prediction is shown as a smooth curve, the gas-gun data are triangles, and the Nova experimental data are squares.
$2 \mathrm{eV}(\sim 20,000 \mathrm{~K})$ is reasonably well represented by this EOS model.

\section{IONIZATION}

Ionization is a more difficult problem. At low gas densities, the Saha chemical equilibrium model for the reaction $\mathrm{D} \rightarrow \mathrm{D}^{+}+\mathrm{e}^{-}$gives an adequate description of ionization [12]. As the density increases, the hydrogen becomes a condensed phase and the electrons occupy bands and cease to act as ideal-gas particles. This requires a Fermi gas treatment, which is quite different from the Saha model. The problem is how to interpolate smoothly between these different models. As a first approximation to the ionization problem, I have chosen to retain the Saha model, but to add a fictitious entropy to the electron gas so that it will behave more like a Thomas-Fermi electron gas at high density. The parameters in the entropy term are adjusted to give optimum agreement with the Thomas-Fermi-Dirac based SESAME EOS [13]. This scheme is the least satisfactory approximation in the new EOS model. It differs from the SESAME model by about $20 \%$ in pressure and energy in the region where the electrons are being excited. Also, a $10 \%$ error is made in the ideal gas high temperature limit in a small density region as a result of the fictitious entropy term.

\section{GLOBAL EOS MODEL}

Adding the cold curve to the thermal solid and fluid EOS models, we obtain a total EOS model which covers the range $0 \leq p \leq 10^{4} \mathrm{~g} / \mathrm{cm}^{3} ; 0 \leq$ $\mathrm{T} \leq 10^{9} \mathrm{~K}$. Starting with a Helmholtz free energy which includes the entropy of mixing of different species, we can compute consistent pressure, internal energy, and entropy functions by differentiating. The final result is a compromise among a number of competing requirements:

1) good fit to experimental isothermal and shock compression data;

2) good fit to theoretical electron band-structure metallic cold curve to $10^{4} \mathrm{~g} / \mathrm{cm}^{3}$;

3) reasonable representation of molecular dissociation in the gas, liquid, and solid states;

4) reasonable representation of atomic ionization in the hot fluid; 
5) correct EOS behavior in the low and high density and temperature limits; and

6) thermodynamic consistency.

Conditions 1,2, and 6 are accurately met; condition 3 is obtained approximately; and conditions 4 and 5 are obtained with low accuracy in certain regions. The total energy surface is shown in Fig. 4.

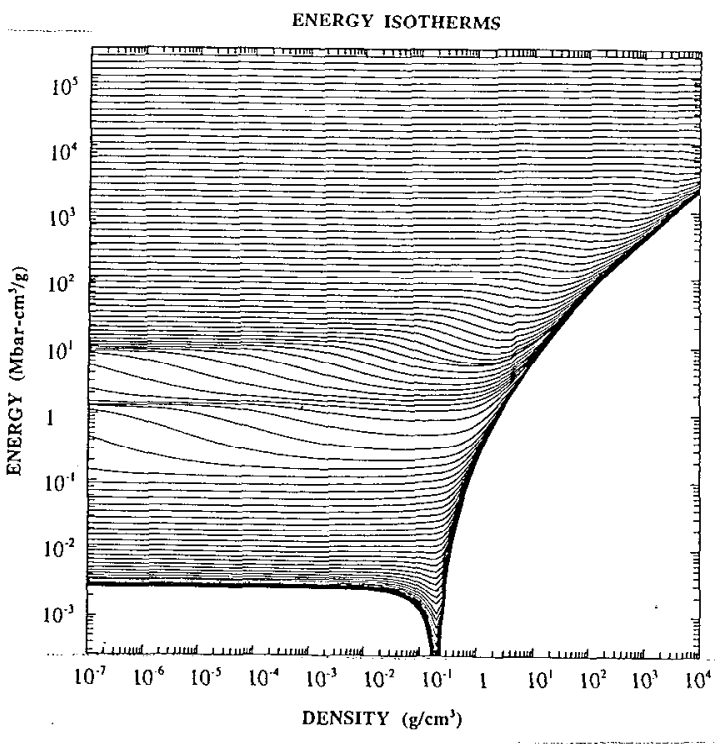

FIGURE 4. The model energy surface for deuterium.

Since the EOS model computations are expensive, the EOS is most efficiently used as a table and stored in an EOS library. Fast lookup and interpolation schemes then allow access to the data. The EOS model has been fitted to the deuterium experimental data. Hydrogen and deuterium-tritium tables can be constructed by simple atomic wcight scaling. This creates small errors near $\mathrm{P}=0$ because of quantum effects, but these errors are negligible for any simulation involving pressures above 1 kilobar.

\section{FUTURE WORK}

It is obvious that we are not yel finished with the global hydrogen EOS problem. We still lack a useful fundamental theory. First-principles quantum Monte-Carlo or finite-temperature LDA calculations are promising, but do not yet have the accuracy and computational efficiency needed to generate good EOS data. Other models are based on more or less good approximations and have obvious limitations in accuracy and $(\rho, \mathrm{T})$ range.

Although hydrogen, with $\mathrm{Z}=1$, is the "simplest" element, condensed hydrogen is in fact a very complex system which challenges the theoretical physicist. It is clear that much more work will be needed to produce truly accurate widerange EOS tables for the hydrogen isotopes.

\section{ACKNOWLEDGMENTS}

I thank Troy Barbee III for the computation of the theoretical LDA cold curve and for useful discussions, and Bob Cauble for providing the deuterium temperature data prior to publication. This work was performed under the auspices of the U.S. Department of Energy by Lawrence Livermore National Laboratory under contract number W-7405ENG-48.

\section{REFERENCES}

1. Young, D. A., Phase Diagrams of the Elements, Univ. California Press, Berkeley, 1991, pp. 268285 .

2. Young, D. A and Corey, F. M., J. Appl. Phys. 78, 3748-3755 (1995).

3. Loubeyre, P., et al., Nature 383, $702-704$ (1996).

4. Barbee III, 'I. W., private communication.

5. Eliezer, S., Ghatak, A., and Hora, H., An Introduction to Equations of State: Theory and Applications, Cambridge Univ. Press, Cambridge, 1986, Chap. 10.

6. More, R., Warren, K. H., Young, D. A., and Zimmerman, G. B., Phys. Fluids 31, 3059-3078 (1988).

7. Nellis, W. J., et al., J. Chem. Phys. 79, 14801486 (1983).

8. Holmes, N. C., Ross, M., and Nellis, W. J., Phys. Rev, B 52, 15835-15845 (1995).

9. DaSilva, L. B., et al., Phys. Rev. Lett. 78, 483486 (1997).

10. Cauble, R., personal communication.

11. Holmes, N. C., Nellis, W. J., and Ross, M., "Sound Velocities in Shocked Liquid Deuterium," in Shock Compression of Condensed Matter - 1997, edited by S. C. Schmidt, et al., AIP Conference Proceedings 429, New York, 1998, pp. 61-64.

12. Ref, 5, Chap. 7.

13. Kerley, G. I., LASL Report LA-4776, January 1972 . 